\title{
Efeito da Linhagem Citoplasmática sobre o Peso ao Nascer e o Ganho Médio Diário na Pré-Desmama em Bovinos da Raça Caracu ${ }^{1}$

\author{
Luciele Cristina Pelicioni' $2,3,4$, Sandra Aidar de Queiroz ${ }^{2,5}$
}

\begin{abstract}
RESUMO - O objetivo do presente estudo foi avaliar o efeito da linhagem citoplasmática sobre as características peso ao nascer (PN) e ganho médio diário pré-desmama (GMD) de, respectivamente, 4.212 e 3844 registros de bezerros de um rebanho da raça Caracu. A linhagem citoplasmática (LC) foi estudada traçando-se o pedigree dos animais até as fêmeas fundadoras do rebanho. Com o intuito de apresentar estimativas de (co)variâncias e parâmetros genéticos para as características PN e GMD, incluindo-se ou não os componentes de efeito materno e de linhagem citoplasmática, foram realizadas análises sob seis diferentes modelos pelo método de máxima verossimilhança restrita livre de derivada. A variância de linhagem citoplasmática nos dois modelos que incluíram esse efeito apresentou-se muito baixa, fornecendo estimativas praticamente nulas, $0,6172 \times 10^{-5}$ e $0,1339 \times 10^{-5} ; 0,62557 \times 10^{-7}$ e $0,49502 \times 10^{-7}$, para PN e GMD, respectivamente. $\mathrm{O}$ efeito de LC pode ser desconsiderado na modelagem das características PN e GMD de bovinos Caracu.
\end{abstract}

Palavras-chave: bovino de dupla aptidão, efeito materno, herança citoplasmática, herdabilidade

\section{Effect of Cytoplasmic Lineage on Birth Weight and Pre Weaning Average Daily Gain of Caracu Cattle Breed}

\begin{abstract}
The objective of this research was to evaluate the effect of cytoplasmic lineage on birth weight (BW) and pre weaning average daily gain (ADG), respectively of 4,212 and 3,844 records of Caracu cattle breed. The cytoplasmic lineage of each cow was traced back to the first female ancestor in the maternal line of its pedigree. The estimates of (co) variances and genetic parameters for BW and ADG including or not the components of maternal and cytoplasmic lineage effects, performed by univariate analyses were evaluated according to six different models using the derivative-free restricted maximum likelihood method. The cytoplasmic lineage variance in the two models that included this effect was very low, $0.6172 \times 10^{-5}$ and $0.1339 \times 10^{-5}, 0.62557 \times 10^{-7}$ and $0.49502 \times 10^{-7}$ for BW and ADG, respectively. The cytoplasmic lineage effect could be neglected in the models of BW and ADG of Caracu cattle.
\end{abstract}

Key Words: cytoplasmic inheritance, dual purpose cattle, heritability, maternal effect

\section{Introdução}

Quando se tem em vista apenas o genótipo de um indivíduo, a contribuição é exatamente $1 / 2$ dos genes do pai e $1 / 2$ dos genes da mãe. Entretanto, a contribuição da mãe para o fenótipo do seu filho se dá não só pela transmissão de efeitos aditivos, ou de efeitos genéticos totais, mas também por meio dos efeitos maternos.

De acordo com HOHENBOKEN (1985), a definição de efeito materno é: "Qualquer contribuição, influência ou impacto sobre o fenótipo de um indivíduo que é atribuído diretamente ao fenótipo de sua mãe".

Em melhoramento, o que se tenta modelar é a contribuição do fenótipo da mãe ou do efeito total da mãe sobre o fenótipo de seu filho.

Diversos mecanismos biológicos explicam os efeitos maternos. Um destes mecanismos que contribuem para o efeito materno total é o DNA extranuclear, que consiste em herança citoplasmática, pois os elementos citoplasmáticos, principalmente a mitocôndria que contém DNA (mtDNA), são herdadas somente da mãe através do citoplasma do óvulo.

A mitocôndria é transmitida intacta de geração a geração, desconsiderando-se as mutações que podem ocorrer no mtDNA. Dessa forma, a herança citoplasmática pode ser obtida traçando-se o pedigree dos animais, pela linha materna, até as fêmeas fundadoras do rebanho.

Um dos primeiros estudos a confirmar a herança

\footnotetext{
1 Parte da Dissertação de Mestrado no programa de Pós-Graduação em Zootecnia, FCAV - UNESP, do primeiro autor, financiada pela FAPESP

2 Departamento de Zootecnia FCAV - UNESP - 14.884-900 - Jaboticabal - SP.

3 Aluna de doutorado do Programa de Pós-Graduação em Zootecnia -Produção Animal-FCAV-UNESP.

${ }^{4}$ Bolsista da FAPESP. E-mail: lucripe@fcav.unesp.br

5 Bolsista do CNPq. E-mail: saquei@fcav.unesp.br
} 
citoplasmática como fonte significativa de variação na produção animal foi o de BELL et al. (1985). Trabalhando com dados da primeira lactação de 4.461 vacas da raça Holandesa e formando linhagens pela identificação das matriarcas do rebanho por pesquisa de pedigree, esses pesquisadores afirmaram que os efeitos de linhagem citoplasmática foram responsáveis por 2,0\% da variância fenotípica para a característica produção total do leite e 3,5\% para porcentagem de gordura do leite.

Para bovinos de corte, as evidências da contribuição da herança citoplasmática nas características de importância econômica são menos conclusivas, com alguns autores (TESS et al., 1987) observando efeitos citoplasmáticos significativos sobre características de crescimento dos bezerros e produção de leite das vacas e outros (NORTHCUTT et al.,1991; TESS e MacNEIL, 1994) concluindo que os efeitos genético-citoplasmáticos não contribuíram para a variação observada no desempenho dos bezerros.

Analisando os efeitos genético-citoplasmáticos sobre crescimento pré-desmama e a produção de leite em bovinos Hereford, TESS et al. (1987) relataram, para os componentes de variância citoplasmática, valores iguais a 2,0;5,0; e 5,0\% da variância fenotípica para peso ao nascer, ganho médio diário pré-desmama e peso aos 205 dias de idade, respectivamente, e $4 \%$ para a produção de leite.

Segundo ROBISON (1981), os efeitos maternos têm sido amplamente discutidos, devido a sua importância econômica na produção animal e possível inclusão em programas de seleção. Os resultados de pesquisas obtidos com bovinos de leite (BELL et al., 1985; HUIZINGA et al., 1986) e suínos (DZPO e WASSMUTH, 1983; TOELLE et al., 1986) revelaram que a influência materna através do citoplasma deve ser considerada nos programas de melhoramento genético animal, tanto para remover esse efeito das estimativas dos valores genéticos quanto como possível fonte extra de variação genética.

O objetivo do presente trabalho foi avaliar o efeito da linhagem citoplasmática (LC) sobre o peso ao nascer (PN) e o ganho médio diário pré-desmama (GMD) dos bezerros de um rebanho da raça Caracu.

\section{Material e Métodos}

Os dados para o presente estudo foram obtidos do arquivo zootécnico da fazenda Chiqueirão, situada no município de Poços de Caldas, MG, que se dedica à criação de bovinos da raça Caracu.
O rebanho Caracu da fazenda Chiqueirão iniciou-se no ano de 1893 , com a aquisição das primeiras matrizes e touros. Os animais foram adquiridos até a década de 40, quando o rebanho foi fechado e passou a produzir seus próprios reprodutores.

Desde a sua formação, o rebanho teve uma criação mais direcionada à exploração leiteira, sendo esta raça considerada de dupla aptidão. A propriedade tem 1800 hectares, aproximadamente, de pastagens que podem ser divididas em duas partes distintas: campo e cultura. O primeiro constitui cerca de $30 \%$ das pastagens, predominando gramíneas nativas da região, enquanto nas áreas de cultura predominam pastagens de gordura (Melinis minutiflora, Pal. de Beauv), elefante (Pennisetum purpureum, Schum) e guiné (Pannicum maximum, Jacq.), além de áreas de cultura de inverno, com azevém (Lolium perene).

A fazenda Chiqueirão possui duas maternidades e dez retiros onde os animais são ordenhados. A ordenha é feita manualmente, duas vezes ao dia, com o bezerro ao pé, e os bezerros ficam separados das mães no intervalo das ordenhas.

O leite proveniente de uma teta é deixado para o bezerro em cada ordenha. Os retiros acomodam de 36 a 75 vacas e o período de lactação é de, aproximadamente, 300 dias, ocasião em que é feita a desmama dos bezerros.

Os animais são criados em regime de pasto nos meses quentes do ano (outubro a maio). Nos meses mais frios, quando as geadas são freqüentes e a maioria das pastagens piora em qualidade, é fornecida silagem de milho para todos os animais.

Para a análise dos dados, foram utilizados 4.212 e 3.844 registros de bezerros nascidos entre 1975 e 1997, para PN e GMD, respectivamente. A formação e editoração dos arquivos foram feitas no programa computacional SAS (1995).

As estações do ano foram divididas em quatro: primavera (setembro, outubro e novembro), verão (dezembro, janeiro e fevereiro), outono (março, abril e maio) e inverno (junho, julho e agosto) pois, na região onde localiza-se a Fazenda Chiqueirão, ocorrem diferenças marcantes entre estes períodos, e a variável grupo contemporâneo (GC) foi criada considerando-se ano e estação do nascimento e sexo do bezerro.

Um programa computacional, em linguagem FORTRAN, foi elaborado para a obtenção das linhagens citoplasmáticas, traçando-se o pedigree dos animais, pela linha materna, até as fêmeas fundadoras do rebanho. A LC foi estudada considerando-se o efeito genético-citoplasmático como aleatório. O GC 
foi incluído em todos os modelos como efeito fixo.

As variáveis $\mathrm{PN}$ e GMD foram corrigidas previamente para a idade da vaca ao parto (PN e GMD) e para a idade do bezerro à desmama (GMD), usando-se fatores de correção calculados para estes efeitos por PELICIONI (2000).

Com o intuito de apresentar estimativas de (co)variâncias e parâmetros genéticos para as características PN e GMD, incluindo-se ou não os componentes de efeito materno, de linhagem citoplasmática e de ambiente permanente, foram realizadas análises sob seis diferentes modelos, a saber:

1으odelo: incluiu os efeitos de GC, genético aditivo direto, genético materno, de linhagem citoplasmática e de ambiente permanente;

20 modelo: incluiu os efeitos de GC, genético aditivo direto, genético materno e de ambiente permanente;

3o modelo: incluiu os efeitos de GC, genético aditivo direto, de linhagem citoplasmática e de ambiente permanente;

4을 modelo: incluiu os efeitos de GC, genético aditivo direto e de ambiente permanente;

5o modelo: incluiu os efeitos de GC e genético aditivo direto;

6-0 modelo: incluiu os efeitos de GC, genético aditivo direto e genético materno.

Em termos matriciais, o modelo 1, o mais completo, pode ser descrito por:

$$
\mathrm{Y}=\mathrm{X} \beta+\mathrm{Za}+\mathrm{Mm}+\mathrm{Dlc}+\mathrm{Wpe}+\mathrm{e}
$$

em que $\mathbf{Y}$ é o vetor de observações; $\boldsymbol{\beta}$, o vetor de efeitos fixos (GC); a, o vetor de efeito genético aditivo direto do animal; $\mathbf{m}$, o vetor de efeito genético aditivo materno; lc, o vetor de efeitos citoplasmáticos, pe é o vetor de efeito ambiental permanente; e, o vetor de efeitos residuais, com $\mathbf{X}, \mathbf{Z}, \mathbf{M}, \mathbf{D}$ e $\mathbf{W}$, sendo as matrizes de incidência associadas aos efeitos apropriados a Y. Para este modelo, $\mathrm{E}[\mathrm{Y}]=\mathrm{X} \beta, \mathrm{E}[\mathrm{a}]=0$, $\mathrm{E}[\mathrm{m}]=0, \quad \mathrm{E}[\mathrm{lc}]=0$ e $\mathrm{E}[\mathrm{e}]=0 ; \quad \operatorname{Var}[\mathrm{a}]=\mathrm{A} \sigma^{2}{ }_{\mathrm{a}}$, $\operatorname{Var}[\mathrm{m}]=\mathrm{A} \sigma_{\mathrm{m}}^{2}, \operatorname{Cov}[\mathrm{a}, \mathrm{m}]=\mathrm{A} \sigma_{\mathrm{am}}, \operatorname{Var}[\mathrm{lc}]=\mathrm{I}_{\mathrm{NC}} \sigma^{2}{ }_{\mathrm{lc}}$, $\operatorname{Var}[\mathrm{pe}]=\mathrm{I}_{\mathrm{NP}} \sigma_{\mathrm{pe}}^{2}, \mathrm{e} \operatorname{Var}[\mathrm{e}]=\mathrm{I}_{\mathrm{N}} \sigma_{\mathrm{e}}^{2}$, NC é o número de linhas maternas; NP, o número de vacas; N, o número de registros; $\mathrm{A}$, o numerador da matriz de parentesco; I, uma matriz identidade; e $\sigma^{2}{ }_{\mathrm{a}}, \sigma^{2}{ }_{\mathrm{m}}, \sigma^{2}{ }_{\mathrm{lc}}$, $\sigma_{\text {pe }}^{2}, \sigma_{\mathrm{e}}^{2}$ e $\sigma_{\mathrm{am}}$, os componentes de variância aditivo direto, aditivo materno, de linhagem citoplasmática, de ambiente permanente, residual e o componente de covariância entre os efeitos aditivo direto e materno, respectivamente. Assume-se que os vetores a e m não são correlacionados com os vetores lc, pe e e.
As estimativas dos componentes de (co)variâncias e dos parâmetros genéticos foram obtidas por máxima verossimilhança restrita livre de derivada (DFRRML "derivative-free restricted maximum likelihood"), utilizando-se o programa computacional MTDFREML ("multiple trait derivative-free restricted maximum likelihood"), descrito por BOLDMAN et al. (1993).O critério de convergência considerado foi de $10^{-9}$.

A herdabilidade do efeito genético total $\left(\mathrm{h}_{\mathrm{T}}^{2}\right)$, isto é, a regressão do genótipo total do animal (direto + materno) sobre o fenótipo, foi calculada como proposto por WILLHAM (1972).

Para verificar o efeito da inclusão da linhagem citoplasmática no modelo de análise, realizou-se o teste de razão de verossimilhança, utilizando-se os modelos 1 (com o efeito de linhagem citoplasmática) e 2 (sem o efeito de linhagem citoplasmática).

Neste teste, segundo FREUND e WALPOLE (1980), define-se $\lambda$ como a razão de verossimilhança calculada como segue:

$$
\lambda=\mathrm{Lc} / \mathrm{Lr}
$$

em que Lc é valor de máxima verossimilhança do modelo completo (modelo 1, com o efeito de linhagem citoplasmática) e Lr, valor de máxima verossimilhança do modelo reduzido (modelo 2 , sem o efeito da linhagem citoplasmática).

Ainda, segundo esses autores, para valor grande de $n$, a distribuição de $-2 \log \lambda$ aproxima-se, em condições gerais, a uma distribuição de chi-quadrado $\left(\chi^{2}\right)$ com 1 grau de liberdade, i.e.:

$$
-2 \log \lambda \sim \chi_{\alpha, v}^{2}
$$

em que: $\alpha$ é nível de significância e $v$, grau de liberdade (1).

O cálculo de - $2 \log \lambda$ pode ser simplificado aplicandose a diferença entre $-2 \log L \mathrm{c}$ e $-2 \log \mathrm{Lr}$, uma vez que:

$$
\begin{gathered}
\lambda=\mathrm{Lc} / \mathrm{Lr}=\mathrm{Lc} \cdot \mathrm{Lr}^{-1} \\
\Rightarrow \log \lambda=\log \mathrm{Lc}-\log \mathrm{Lr} \\
\Rightarrow-2 \log \lambda=(-2 \log \mathrm{Lc})-(-2 \log \mathrm{Lr})
\end{gathered}
$$

Assim, quando $-2 \log \lambda \geq \chi_{\alpha, v}^{2}$, pode-se afirmar que o efeito é significativo.

\section{Resultados e Discussão}

A média observada para peso ao nascer (PN) foi $31,98 \mathrm{~kg}$, variando de 18,00 a 48,00 kg. Esta média está próxima à obtida, no mesmo rebanho, por AMARAL (1990), que foi de $31,62 \mathrm{~kg}$.

As estimativas dos componentes de (co)variância e dos parâmetros genéticos para PN são mostradas na Tabela 1. Na Tabela 2 são apresentadas as estimativas dos componentes de variância como por- 
centagem da variância fenotípica total.

As estimativas de $\sigma_{\mathrm{a}}{ }^{2}$ e $\mathrm{h}_{\mathrm{a}}^{2}$ obtidas sob os modelos 1 e 2 foram as mais altas, quando comparadas com as obtidas sob os outros modelos.

Ignorando-se o efeito genético materno (modelos 3 e 4), houve relativo aumento da $\sigma_{\mathrm{pe}}{ }^{2}$ e da $\sigma_{\mathrm{e}}{ }^{2} \mathrm{em}$ relação aos modelos 1 e 2 , reduzindo as estimativas de $\sigma_{\mathrm{a}}^{2} \mathrm{eh}_{\mathrm{a}}^{2}$, quando comparadas aos demais modelos.

Os modelos 1 e 2 foram muito semelhantes, sugerindo, portanto, pouca influência do efeito de linhagem citoplasmática sobre peso ao nascer.

No modelo 4, que incluiu apenas os efeitos genético aditivo direto e de ambiente permanente, verifica-se menor valor de $\sigma_{\mathrm{a}}{ }^{2}$, em relação aos modelos 1 , 2,5 e 6 , com a estimativa de $\sigma_{\mathrm{pe}}^{2}$ maior que nos demais modelos, indicando que a $\sigma_{\mathrm{pe}}{ }^{2}$ sob esse modelo (4) continha, além da variação devida ao efeito de ambiente permanente, também, a variação causada pelo componente de efeito materno.

Comparando-se os modelos 1 e 3 , verifica-se que a estimativa de $\sigma_{\mathrm{a}}^{2}$ no modelo 3 diminuiu em relação ao modelo 1 , já as estimativas de $\sigma_{\mathrm{pe}}{ }^{2}$ e $\sigma_{\mathrm{e}}^{2}$ aumentaram em relação ao modelo 1 , demonstrando a importância da inclusão do efeito materno no modelo.
$\mathrm{Na}$ verdade, parte da $\sigma_{\mathrm{m}}{ }^{2}$ e $\sigma_{\mathrm{a}}^{2}$ encontradas no modelo 3 ficou contida nas $\sigma_{\mathrm{pe}}{ }^{2} \mathrm{e} \sigma_{\mathrm{e}}{ }^{2}$.

Os modelos 3 e 4 foram idênticos, confirmando a pequena ou nula contribuição do efeito de linhagem citoplasmática.

A estimativa de $\sigma_{\mathrm{e}}^{2}\left(8,44793 \mathrm{~kg}^{2}\right)$ obtida no modelo 5 apresentou-se maior em relação ao modelo $1\left(7,83238 \mathrm{~kg}^{2}\right)$, verificando-se, também, que parte da $\sigma_{\mathrm{e}}^{2}$ obtida no modelo 5 foi explicada pelos efeitos de ambiente permanente, materno e linhagem citoplasmática no modelo 1.

Comparando os modelos 1 e 6 , a estimativa de $\sigma_{\mathrm{m}}{ }^{2}$ no modelo 6 aumentou em relação ao modelo 1 . $\mathrm{Na}$ verdade, parte da $\sigma_{\mathrm{m}}{ }^{2}$ obtida no modelo 6 $\left(1,05943 \mathrm{~kg}^{2}\right)$ foi explicada pelos efeitos de ambiente permanente no modelo 1 .

Comparando-se os modelos 4 (com efeito de ambiente permanente) e 5 (sem efeito de ambiente permanente), a estimativa de $\sigma_{\mathrm{a}}^{2}$ no modelo 4 diminuiu em relação ao modelo 5, demonstrando que parte da $\sigma_{\mathrm{a}}^{2}$ obtida em $5\left(3,50636 \mathrm{~kg}^{2}\right)$ foi explicada pelo efeito de ambiente permanente da mãe (modelo 4). A inclusão deste reduziu a estimativa de $\mathrm{h}_{\mathrm{a}}{ }^{2}$ e, conseqüentemente, de $\mathrm{h}_{\mathrm{T}}{ }^{2}$, em 10,12\% em relação ao modelo 5 .

Tabela 1 - Estimativas dos componentes de (co)variância $\left(\mathrm{em} \mathrm{kg}^{2}\right)$ e parâmetros genéticos para o peso ao nascer de bezerros da raça Caracu

Table 1 - Estimates of (co)variances components (in $\mathrm{kg}^{2}$ ) and genetic parameters for birth weight of Caracu cattle breed

\begin{tabular}{|c|c|c|c|c|c|c|}
\hline \multirow{2}{*}{$\begin{array}{l}\text { Componentes } \\
\text { Components }\end{array}$} & \multicolumn{5}{|c|}{$\begin{array}{l}\text { Modelos } \\
\text { Models }\end{array}$} & \multirow[b]{2}{*}{6} \\
\hline & 1 & 2 & 3 & 4 & 5 & \\
\hline$\sigma_{\mathrm{a}}^{2}$ & 3,62107 & 3,62107 & 3,13417 & 3,13513 & 3,50636 & 3,57531 \\
\hline$\sigma_{\mathrm{m}}^{2}$ & 0,87046 & 0,87046 & - & - & - & 1,05943 \\
\hline$\sigma_{\mathrm{am}}$ & $-0,64667$ & $-0,64667$ & - & - & - & $-0,65282$ \\
\hline$\sigma_{l c}^{2}$ & $0,6172 \times 10^{-5}$ & - & $0,1339 \times 10^{-5}$ & - & - & - \\
\hline$\sigma_{\mathrm{pe}}^{2}$ & 0,25835 & 0,25835 & 0,61551 & 0,61649 & - & - \\
\hline$\sigma_{\mathrm{e}}^{2}$ & 7,83238 & 7,83238 & 8,14269 & 8,14035 & 8,44793 & 7,95625 \\
\hline$\sigma_{p}^{2}$ & 11,93561 & 11,93560 & 11,89238 & 11,89197 & 11,95429 & 11,93817 \\
\hline$h^{2}{ }_{a}^{r}$ & 0,3033 & 0,3033 & 0,2635 & 0,2636 & 0,2933 & 0,2994 \\
\hline$h^{2}{ }_{m}$ & 0,0729 & 0,0729 & - & - & - & 0,0887 \\
\hline$h^{2}{ }_{T}$ & 0,2585 & 0,2585 & 0,2635 & 0,2636 & 0,2933 & 0,2618 \\
\hline $\mathrm{r}_{\mathrm{am}}$ & $-0,36$ & $-0,36$ & - & - & - & $-0,34$ \\
\hline
\end{tabular}

$\sigma^{2}, \sigma_{a}^{2}, \sigma^{2}{ }_{m}, \sigma_{\text {lc }}^{2} \sigma_{p e}^{2}, \sigma_{p}^{2}, \sigma_{a m}, h^{2}{ }_{a}, h^{2}{ }_{m}, h^{2}{ }_{T}, r_{a m}$ e -2log $\lambda=$ componentes de variância residual, aditivo direto, aditivo materno, de linhagem citoplasmática, de ambiente permanente e fenotípica total, componente de covariância entre os efeitos aditivos direto e materno, herdabilidade do efeito direto, herdabilidade do efeito materno, herdabilidade total, correlação genética entre os efeitos direto e materno e -2 logaritmo da razão de verossimilhança obtido pelo teste de razão de verossimilhança, respectivamente. ${ }^{\text {ns }}=$ não significativo $(P>0,05)$.

$\sigma^{2}{ }_{e}, \sigma^{2}{ }_{a}, \sigma^{2}{ }_{m}, \sigma_{l c}^{2} \sigma^{2}{ }_{p e}, \sigma^{2}{ }_{p}, \sigma_{a m}, h^{2}{ }_{a}, h^{2}{ }_{m}, h^{2}{ }_{T}, r_{a m} e-2 \log \lambda=$ residual variance, direct additive genetic variance, maternal additive genetic variance, cytoplasmic lineage variance, maternal permanentenvironmental variance, phenotypic variance, covariance between directand maternal effects, directheritability, maternal heritability, total heritability and genetic correlation between direct and maternal effects and -2 log likelihood ratio, respectively. ${ }^{n s}=n o t$ significant $(P>05)$. 
Rev. bras. zootec.

Tabela 2 - Estimativas dos componentes de variância, para o peso ao nascer de bezerros da raça Caracu, expressas como porcentagem da variância fenotípica total

Table 2 - Variance components estimates for birth weight of Caracu cattle breed, expressed as a percentage of total phenotipic variance

\begin{tabular}{|c|c|c|c|c|c|c|}
\hline \multirow{2}{*}{$\begin{array}{l}\text { Componentes } \\
\text { Components }\end{array}$} & \multicolumn{6}{|c|}{$\begin{array}{l}\text { Modelos } \\
\text { Models }\end{array}$} \\
\hline & 1 & 2 & 3 & 4 & 5 & 6 \\
\hline$\sigma_{\mathrm{a}}^{2}(\%)$ & 30,33 & 30,33 & 26,35 & 26,36 & 29,33 & 29,94 \\
\hline$\sigma^{2}{ }_{m}(\%)$ & 7,29 & 7,29 & - & - & - & 8,87 \\
\hline$\sigma_{1 \mathrm{c}}^{2}(\%)$ & $5,17 \times 10^{-5}$ & - & $1,12 \times 10^{-5}$ & - & - & - \\
\hline$\sigma_{\mathrm{pe}}^{2}(\%)$ & 2,16 & 2,16 & 5,17 & 5,18 & - & - \\
\hline$\sigma^{2} \sigma^{2} \quad(\%)$ & $\begin{array}{r}65,32 \\
100,00\end{array}$ & $\begin{array}{r}65,62 \\
10000\end{array}$ & $\begin{array}{r}68,47 \\
10000\end{array}$ & $\begin{array}{r}68,45 \\
10000\end{array}$ & 70,67 & 66,64 \\
\hline$\sigma_{p}^{2}(\%)$ & 100,00 & 100,00 & 100,00 & 100,00 & 100,00 & 100,00 \\
\hline
\end{tabular}

$\sigma^{2}{ }_{e}, \sigma_{a}^{2}, \sigma_{m}^{2}, \sigma_{\text {lc }}^{2} \sigma_{p e}^{2}$ e $\sigma_{p}^{2}=$ componentes de variância residual, aditivo direto, aditivo materno, de linhagem citoplasmática, de ambiente permanente e fenotípica total, respectivamente

$\sigma^{2}{ }_{e}, \sigma_{a}^{2}, \sigma_{m}^{2}, \sigma_{l c}^{2} \sigma_{p e}^{2}, \sigma_{p}^{2}=$ residual variance, direct additive genetic variance, maternal additive genetic variance, cytoplasmic lineage variance, maternal permanent environmental variance, phenotypic variance, respectively

Ignorando-se o efeito de ambiente permanente (modelos 5 e 6), houve pequeno aumento na $\sigma_{\mathrm{p}}^{2}$ e $\sigma_{\mathrm{e}}^{2}$, sugerindo que a inclusão deste efeito proporcionou melhor correção das observações, no que diz respeito aos efeitos ambientais da parte fixa do modelo.

Comparando-se os modelos 5 e 6 , percebe-se que houve aumento na $\sigma_{\mathrm{a}}{ }^{2}$ e redução na $\sigma_{\mathrm{e}}{ }^{2}$ no modelo 6 em relação às obtidas no modelo 5 , sugerindo que parte da $\sigma_{\text {pe }}^{2}$ foi explicada pelo efeito materno e outra parte embutida na estimativa de $\sigma_{\mathrm{a}}{ }^{2}$.

Apesar de o modelo 6 não incluir o efeito de ambiente permanente, a variação devida a este efeito ficou, na sua maioria, contida na $\sigma_{\mathrm{m}}{ }^{2}$, explicando o fato de a estimativa de $\mathrm{h}_{\mathrm{T}}{ }^{2}$ obtida sob este modelo ser semelhante às obtidas sob os modelos 1,2, 3 e 4, que incluíram o efeito de ambiente permanente.

Comparando-se os modelos 1 e 2, percebe-se que as estimativas de $\sigma_{\mathrm{m}}^{2}$ e $\sigma_{\mathrm{am}}$ reduziram a $\sigma_{\mathrm{pe}}{ }^{2} \mathrm{e}$ a não-inclusão do efeito genético materno nos modelos 3 e 4 promoveu aumento na $\sigma_{\text {pe }}^{2}$ e redução na $\sigma_{\mathrm{a}}{ }^{2}$. Desconsiderando-se o efeito de ambiente permanente (modelos 5 e 6), incluindo-se ou não o efeito genético materno, percebe-se aumento na $\sigma_{\mathrm{a}}{ }^{2}$.

Nos dois modelos que incluíram o efeito aleatório de linhagem citoplasmática, a $\sigma_{\mathrm{lc}}^{2}$ apresentou-se muito baixa, fornecendo estimativas, praticamente, nulas $\left(0,6172 \times 10^{-5}\right.$ e $0,1339 \times 10-5 \mathrm{~kg}^{2}$, modelos 1 e 3 , respectivamente). Tais estimativas aproximam-se da encontrada por ALENCAR et al. (1998), que, trabalhando com 4722 informações de pesos ao nascer de bezerros da raça Canchim, verificaram estimativa de componente de variância para linhagem citoplasmática igual a 0,00.
Verifica-se, na Tabela 1 , que o valor de $-2 \log \lambda$ calculado mostrou-se não-significativo a $5 \%$ de probabilidade. Assim, os resultados encontrados, neste estudo, indicam pequena ou nula contribuição do efeito de linhagem citoplasmática para a característica peso ao nascer.

A média observada para a característica GMD foi de $0,768 \mathrm{~kg}$, com variação de 0,281 a $1,958 \mathrm{~kg}$. Tal média está próxima à obtida, no mesmo rebanho, por AMARAL (1990), 0,630 kg.

As estimativas dos componentes de (co)variância e dos parâmetros genéticos para o ganho médio diário de peso pré-desmama são apresentadas na Tabela 3 e as estimativas dos componentes de variância como porcentagem da variância fenotípica total, na Tabela 4.

A análise sob o modelo mais completo (modelo 1) forneceu valor da estimativa de $\sigma_{\mathrm{a}}{ }^{2}$ menor que as obtidas sob os demais modelos. Ignorando-se o efeito genético materno (modelo 3), houve aumento da $\sigma_{\mathrm{pe}}^{2}$ em relação aos modelos 1,2 e 4 . Os modelos 1 e 2 foram muito semelhantes, indicando, assim, pequena ou nula contribuição do efeito de linhagem citoplasmática.

Comparando-se os modelos 1,2, 3 e 4, observam-se estimativas de $\sigma_{\mathrm{a}}^{2}$ semelhantes. Verifica-se, também, que parte da $\sigma_{\mathrm{pe}}{ }^{2}$ (modelos 3 e 4 ) foi explicada pelo efeito genético materno (modelo 1 e 2).

Verifica-se que os modelos 3 e 4 , também, foram muito semelhantes, confirmando a pequena ou nula contribuição do efeito citoplasmático para a característica GMD. Observa-se, ainda, que o efeito genético materno nesses modelos ficou contido na estimativa de $\sigma^{2}$ pe 
Tabela 3 - Estimativas dos componentes de (co)variância $\left(\mathrm{em} \mathrm{kg}^{2}\right)$ e parâmetros genéticos para o ganho médio diário de peso pré-desmama de bezerros da raça Caracu

Table 3 - Estimates of (co)variances components (in $\mathrm{kg}^{2}$ ) and genetic parameters for preweaning average daily gain of Caracu cattle breed

\begin{tabular}{|c|c|c|c|c|c|c|}
\hline \multirow{2}{*}{$\begin{array}{l}\text { Itens } \\
\text { Components }\end{array}$} & \multicolumn{5}{|c|}{$\begin{array}{l}\text { Modelos } \\
\text { Models }\end{array}$} & \multirow[b]{2}{*}{6} \\
\hline & 1 & 2 & 3 & 4 & 5 & \\
\hline$\sigma_{a}^{2}$ & 0,00165 & 0,00166 & 0,00167 & 0,00168 & 0,00190 & 0,00165 \\
\hline$\sigma_{\mathrm{m}}^{2}$ & 0,00042 & 0,00041 & - & - & - & 0,00054 \\
\hline$\sigma_{\mathrm{am}}$ & $-0,00015$ & $-0,00015$ & - & - & - & $-0,00016$ \\
\hline$\sigma_{l c}^{2}$ & $0,62557 \times 10-7$ & - & $0,49502 \times 10-7$ & - & - & - \\
\hline$\sigma_{\mathrm{pe}}^{2}$ & $0,18527 \times 10-3$ & $0,18655 \times 10-3$ & $0,43856 \times 10-3$ & $0,43436 \times 10-3$ & - & - \\
\hline$\sigma_{\mathrm{e}}^{2}$ & 0,00779 & 0,00780 & 0,00778 & 0,00780 & 0,00804 & 0,00789 \\
\hline$\sigma_{p}^{2}$ & 0,00988 & 0,00991 & 0,00989 & 0,00991 & 0,00994 & 0,00991 \\
\hline $\mathrm{h}^{2}{ }_{\mathrm{a}}$ & 0,1670 & 0,1675 & 0,1688 & 0,1695 & 0,1911 & 0,1664 \\
\hline $\mathrm{h}_{\mathrm{m}}^{2}$ & 0,0425 & 0,0413 & - & - & - & 0,0545 \\
\hline $\mathrm{h}_{\mathrm{T}}^{2}$ & 0,1654 & 0,1654 & 0,1688 & 0,1695 & 0,1911 & 0,1695 \\
\hline $\mathrm{r}_{\mathrm{am}}$ & $-0,18$ & $-0,18$ & - & - & - & $-0,17$ \\
\hline
\end{tabular}

$\sigma^{2}{ }_{e}, \sigma_{a}^{2}, \sigma^{2}{ }_{m}, \sigma_{\text {lc }}^{2} \sigma_{p e}^{2}, \sigma^{2}, \sigma_{a m}, h^{2}{ }_{a}, h^{2}{ }_{m}, h^{2}{ }_{T}, r_{a m}$ e $-2 \log \lambda=$ componentes de variância residual, aditivo direto, aditivo materno, de linhagem citoplasmática, de ambiente permanente e fenotípica total, componente de covariância entre os efeitos aditivos direto e materno, herdabilidade do efeito direto, herdabilidade do efeito materno, herdabilidade total, correlação genética entre os efeitos direto e materno e -2 logaritmo da razão de verossimilhança obtido pelo teste de razão de verossimilhança, respectivamente. ns = não significativo $(P>0,05)$.

$\sigma_{e}^{2}, \sigma_{a}^{2}, \sigma_{m}^{2}, \sigma_{l c}^{2} \sigma_{p e}^{2}, \sigma_{p}^{2}, \sigma_{a m}, h_{a}^{2}, h_{m}^{2}, h^{2}{ }_{T}, r_{a m}$ e-2log $\lambda=$ residual variance, direct additive genetic variance, maternal additive genetic variance, cytoplasmic lineage variance, maternal permanentenvironmental variance, phenotypic variance, covariance between directand maternal effects, directheritability, maternal heritability, total heritability and genetic correlation between direct and maternal effects and -2 log likelihood ratio, respectively. $n s=n o t$ significant $(P>05)$.

As estimativas de $\sigma_{a}^{2}$ e $h_{a}^{2}$ obtidas sob o modelo 5 foram, substancialmente, mais altas quando comparadas com as obtidas sob os outros modelos. Observa-se, comparando-se os modelos 1 e 5 , que parte da $\sigma_{\mathrm{a}}^{2}$ e $\sigma_{\mathrm{e}}^{2}$ obtidas no modelo 5 foi explicada pelas estimativas de $\sigma_{\mathrm{m}}{ }^{2}, \sigma_{\mathrm{am}}$ e $\sigma_{\mathrm{pe}}{ }^{2}$ encontradas no modelo 1 .

Os modelos 1 e 6 apresentaram estimativas de $\sigma_{\mathrm{a}}^{2}$ e $\sigma_{\mathrm{e}}^{2}$ semelhantes, demonstrando que parte da $\sigma_{\mathrm{m}}^{2}$ obtida no modelo 6 foi explicada pela estimativa de $\sigma_{\mathrm{pe}}^{2}$ no modelo 1 .

Quando somente os efeitos genético aditivo direto e de ambiente permanente da mãe foram incluídos (modelo 4), a estimativa de $\sigma^{2}$ a diminuiu em relação ao modelo 5 (sem o efeito de ambiente permanente). $\mathrm{Na}$ verdade, parte da $\sigma^{2}$ a obtida sob o modelo 5 $\left(0,00190 \mathrm{~kg}^{2}\right)$ foi explicada pelo efeito de ambiente permanente da mãe (modelo 4). A inclusão do efeito de ambiente permanente reduziu a estimativa de $h_{a}^{2}$ e, conseqüentemente, de $\mathrm{h}_{\mathrm{T}}{ }^{2}$, em relação ao modelo 5 .

Comparando-se os modelos 5 e 6 , observa-se a importância da inclusão do efeito materno no modelo, pois parte da $\sigma_{\mathrm{a}}^{2}$ e $\sigma_{\mathrm{e}}^{2}$ obtidas no modelo 5 é explicada pela $\sigma_{\mathrm{m}}^{2}$ no modelo 6 .
Os modelos que incluíram o efeito aleatório de linhagem citoplasmática apresentaram $\sigma_{\mathrm{lc}}{ }^{2}$ muito baixa, fornecendo estimativas, praticamente, nulas $\left(0,625571 \times 10^{-5}\right.$ e $0,495024 \times 10^{-5}$ para os modelos 1 e 3 , respectivamente). A pouca importância desse efeito pode ser verificada, na Tabela 2, pelo valor de $-2 \log \lambda$ calculado para a característica GMD que mostrou-se não significativo a 5\% de probabilidade.

A covariância entre efeitos direto e materno $\left(\sigma_{\mathrm{am}}\right)$ é um assunto muito discutido em trabalhos científicos. Nos relatos encontrados na literatura, predominam estimativas de covariância negativas entre os efeitos direto e materno. Neste trabalho, as estimativas de $\sigma_{\text {am }}$ para PN e GMD (modelos 1, 2 e 6) foram negativas, fornecendo estimativas baixas de correlação $\left(\mathrm{r}_{\mathrm{am}}\right)$ entre estes efeitos, concordando com as correlações obtidas por ELER et al. (1995 e 1996), $-0,13$ e $-0,22$, respectivamente, para a característica GMD.

De acordo com Schaeffer (1996), citado por FRIES e ALBUQUERQUE (1998), caso não existam filhos suficientes com registros próprios de produção e, também, com progênies medidas, isto é, indivíduos que tiveram efeito direto medido, quando 
Rev. bras. zootec.

Tabela 4 - Estimativas dos componentes de variância, para o ganho médio diário de peso pré-desmama de bezerros da raça Caracu, expressas como porcentagem da variância fenotípica total

Table 4 - Variance components estimates for average daily gain of Caracu cattle breed expressed as a percentage of total phenotipic variance

\begin{tabular}{|c|c|c|c|c|c|c|}
\hline \multirow{2}{*}{$\begin{array}{l}\text { Componentes } \\
\text { Components }\end{array}$} & \multicolumn{5}{|c|}{$\begin{array}{c}\text { Modelos } \\
\text { Models }\end{array}$} & \multirow[b]{2}{*}{6} \\
\hline & 1 & 2 & 3 & 4 & 5 & \\
\hline$\sigma_{\mathrm{a}}^{2}(\%)$ & 16,70 & 16,75 & 16,88 & 16,95 & 19,11 & 16,64 \\
\hline$\sigma_{m}^{2}(\%)$ & 4,25 & 4,13 & - & - & - & 5,45 \\
\hline$\sigma^{2}{ }_{l c}(\%)$ & $6,33 \times 10-4$ & - & $5,00 \times 10-4$ & - & - & - \\
\hline$\sigma_{\mathrm{pe}}^{2}(\%)$ & 1,87 & 1,88 & 4,43 & 4,38 & - & - \\
\hline$\sigma_{\mathrm{e}}^{2}(\%)$ & 78,84 & 78,70 & 78,66 & 78,70 & 80,88 & 79,61 \\
\hline$\sigma_{p}^{2}(\%)$ & 100,00 & 100,00 & 100,00 & 100,00 & 100,00 & 100,00 \\
\hline
\end{tabular}

$\sigma_{e}^{2}, \sigma_{a}^{2}, \sigma_{m}^{2}, \sigma_{l c}^{2} \sigma_{p e}^{2}$ e $\sigma_{p}^{2}=$ componentes de variância residual, aditivo direto, aditivo materno, de linhagem citoplasmática, de ambiente permanente, fenotípica total, respectivamente.

$\sigma_{e}^{2}, \sigma^{2}{ }_{a}, \sigma_{m}^{2}{ }_{m} \sigma_{l c}^{2} \sigma_{p e}^{2}, \sigma_{p}^{2}=$ residual variance, direct additive genetic variance, maternal additive genetic variance, cytoplasmic lineage variance, maternal permanent environmental variance, phenotypic variance, respectively.

eram bezerros, e depois tiveram efeito materno medido, também, quando se tornaram mães, a estimativa da covariância é um problema. Neste trabalho, fez-se uso de um conjunto de dados com animais nascidos desde 1975 pertencentes ao mesmo rebanho e, assim, grande parte dos indivíduos foi medida tanto como produto quanto como mãe. Dessa forma, esse problema não foi tão evidente.

As estimativas de herdabilidade materna $\left(\mathrm{h}_{\mathrm{m}}\right)$, encontradas para PN e GMD, apresentaram-se baixas, demonstrando a pouca influência do efeito materno sobre estas características, sugerindo que este tem menor importância que o efeito direto nesta fase da vida do animal. Assim como a $h_{m}$, os pequenos valores estimados para $\sigma_{\mathrm{m}}^{2}$ e $\sigma_{\mathrm{pe}}{ }^{2}$ obtidos para PN e GMD podem ser explicados, provavelmente, pelo manejo adotado pela fazenda, pois a ordenha é feita manualmente, duas vezes ao dia, com o bezerro ao pé, e os bezerros ficam separados das mães no intervalo entre elas, sendo o leite proveniente de apenas uma teta deixado para o bezerro em cada ordenha. Em síntese, os bezerros permanecem pouco tempo com as mães e não têm grande quantidade de leite disponível para mamar.

$\mathrm{Na}$ análise geral dos modelos, é possível verificar a influência relevante do efeito materno sobre o peso ao nascer e o ganho médio diário de peso prédesmama, como era de se esperar, e a pequena ou nula contribuição do efeito de linhagem citoplasmática sobre estas características.

\section{Conclusões}

Houve pequena, mas relevante, contribuição do efeito materno para PN e GMD.

$\mathrm{O}$ modelo 2, na análise das características $\mathrm{PN}$ e GMD, apresentou-se como o melhor, por ser mais simplificado que o modelo 1 (modelo completo) e fornecer estimativas semelhantes.

Para as características PN e GMD, a não-inclusão do efeito de ambiente permanente no modelo induz a super estimação do efeito materno.

O efeito de linhagem citoplasmática mostrou ser pouco importante na determinação de PN e GMD, no rebanho Caracu estudado.

\section{Agradecimento}

Ao zootecnista M.S. André Carvalho Dias (Fazenda Chiqueirão), pela cessão dos dados, e à FAPESP, pelo suporte financeiro.

\section{Referências Bibliográficas}

ALENCAR, M.M., TREMATORE, R.L., BARBOSA, P.F. et al. 1998. Estudo da influência citoplasmática sobre pesos em bovinos da raça Chanchim. R. Bras. Zootec., 27(2):272-276.

AMARAL, M. O. Efeito da endogamia sobre o peso ao nascer e à desmama de bezerros da raça Caracu. Jaboticabal, SP: FCAV, 1990. 53 p. Monografia (Graduação em Zootecnia) Faculdade de Ciências Agrárias e Veterinárias, Uiversidade Estadual Paulista, 1990. 
BELL, B.R., McDANIEL, B.T., ROBISON, O.W. 1985. Effects of cytoplasmic inheritance on production traits of dairy cattle. J. Dairy Sci., 68:2038.

BOLDMAN, K.G., KRIESE, L.A., VAN VLECK, L.D. et al. 1993. A Manual for Use of MTDFREML. USDA-ARS. Clay Center, NE. 120p.

DZPO, V.V., WASSMUTH, R. 1983. Mitochondrialer stoffwechsel und heterotische effekte beim schwein. Ergebnisse eines reziproken kreuzungs-versuches. II. Atmungsaktivitat und oxydative phosphorylierung in herz, leber und hodenmitochondrien. Z. Tierz. Zuchtungsbiol, 100:280.

ELER, J.P., VAN VLECK, L.D., FERRAZ, J.B.S. et al. 1995. Estimation of variances due to direct and maternal effects for growth traits of Nellore cattle. J. Anim. Sci., 73:3253-3258.

ELER, J.P., FERRAZ, J.B.S., SILVA, P.R. Estimação simultânea de parâmetros genéticos para características de importância econômica na raça Nelore, com a utilização de modelos animais. In: REUNIÃO ANUAL DA SOCIEDADE BRASILEIRA DE ZOOTECNIA, 33, 1996, Fortaleza. Anais... Fortaleza: UFC, 1996. p.99-101.

FREUND, J. F., WALPOLE, R.E. 1980. Mathematical statistics, 3.ed. New Jersey: Prentice-Hall. 548p.

FRIES, L.A., ALBUQUERQUE, L.G. de. 1998. Pressuposições e restrições dos modelos animais com efeitos maternos em gado de corte. In: SBEt (Ed.) Comportamento materno em mamíferos: bases teóricas e aplicações aos ruminantes domésticos. p.179-214.

HOHENBOKEN, W.D. 1985. Maternal effects. In: CHAPMAN, A.B. (Ed.) World Animal Science A4: General and Quantitative genetics. Amsterdam: Elsevier. p.135-149.

HUIZINGA, H.A., KORVER, S., McDANIEL et al. 1986. Maternal effects due to cytoplasmic inheritance in dairy cattle. Livest. Prod. Sci., 15:11.
NORTHCUTT, S.L., WILLHAM, R.L., WILSON, D.E. 1991. Genetic parameters for nuclear and nonnuclear inheritance in three synthetic lines of beef cattle differing in mature size. J. Anim. Sci., 69(12):4745- 4753.

PELICIONI, L. C. Polinômios segmentados no ajuste de idade e efeitos da linhagem citoplasmática para características de crescimento e produção de leite em bovinos da raça Caracu. Jaboticabal, SP: FCAV 2000. 122 p. Dissertação (Mestrado em Zootecnia) - Faculdade de Ciências Agrárias e Veterinárias, Universidade Estadual Paulista, 2000.

ROBISON, O.W. 1981. The influence of maternal effects on the efficiency of selection, A review.Livest. Prod. Sci., 8(2):121-137.

SAS, 1995. Institute Inc. SAS Language Guide, v. 6.03 Edition, Care, NC: SAS INSTITUTE INC. 530p.

TESS, M.W., MacNEIL, M. D. 1994. Evaluation of cytoplasmic genetics effects in Miles City Line 1 Hereford cattle. J. Anim. Sci., 72(4):851-856.

TESS, M.W., REODECHA, C., ROBISON, O.W. 1987. Cytoplasmic genetics effects on preweaning growth and milk yield in Hereford cattle. J. Anim. Sci., 65(3):675-684.

TOELlE, V.D., McDANIEL, ROBISON, O.W. 1986. Cytoplasmic effects in swine. J. Anim. Sci., 63(Suppl. 1):203.

WILLHAM, R. L. 1972. The role of maternal effects in animal breeding: III-Biometrical aspects of maternal effects in animals. J. Anim. Sci., 35:1288-1302.

Recebido em: 16/06/00

Aceito em: 08/09/00 